Article

\title{
Propagation and Separation of Downslope Gravity Currents over Rigid and Emergent Vegetation Patches in Linearly Stratified Environments
}

\author{
Ying-Tien Lin ${ }^{1,2} \mathbb{D}$, Yi-Qi Ye ${ }^{1}$, Dong-Rui Han $^{1}$ and Yu-Jia Chiu ${ }^{3,4, *}$ \\ 1 Institute of Port, Coastal and Offshore Engineering, Ocean College, Zhejiang University, \\ Zhoushan 316021, China; kevinlin@zju.edu.cn (Y.-T.L.); 22034168@zju.edu.cn (Y.-Q.Y.); \\ 21734097@zju.edu.cn (D.-R.H.) \\ 2 Engineering Research Center of Oceanic Sensing Technology and Equipment, Zhejiang University, \\ Ministry of Education, Zhoushan 316021, China \\ 3 Department of Harbor and River Engineering, National Taiwan Ocean University, Keelung 202301, Taiwan \\ 4 Center of Excellence for Ocean Engineering, National Taiwan Ocean University, Keelung 202301, Taiwan \\ * Correspondence: yujiachiu@ntu.edu.tw or yjchiu@mail.ntou.edu.tw
}

check for updates

Citation: Lin, Y.-T.; Ye, Y.-Q.; Han, D.-R.; Chiu, Y.-J. Propagation and Separation of Downslope Gravity Currents over Rigid and Emergent Vegetation Patches in Linearly Stratified Environments. J. Mar. Sci. Eng. 2022, 10, 308. https:// doi.org/10.3390/jmse10030308

Academic Editor:

Evangelos Keramaris

Received: 20 January 2022

Accepted: 20 February 2022

Published: 22 February 2022

Publisher's Note: MDPI stays neutral with regard to jurisdictional claims in published maps and institutional affiliations.

Copyright: (C) 2022 by the authors. Licensee MDPI, Basel, Switzerland. This article is an open access article distributed under the terms and conditions of the Creative Commons Attribution (CC BY) license (https:// creativecommons.org/licenses/by/ $4.0 /)$.

\begin{abstract}
Large eddy simulation (combined with the mixture model) and laboratory experiment were used to investigate the impact of emergent and rigid vegetation on the dynamics of downslope gravity currents in stratified environments. The reliability of the numerical model was assessed with the corresponding laboratory measurements. The results show that the vegetation cylinders lead to severe lateral non-uniformity of the current front, causing more evident lobe and cleft structures. In stratified environments, the smaller driving force leads to less propagating velocity until the current separates from the slope. The transition point (from acceleration to deceleration phases) of current velocity appears earlier as the vegetation becomes denser. The peak value of the bulk entrainment coefficient $E_{\text {buik }}$ is inversely proportional to the vegetation density, while the final converged value of $E_{b u i k}$ is proportional to the vegetation density. Vegetation patches make the degree of fluctuation of the instantaneous entrainment coefficient $E_{\text {inst }}$ more intense, and even negative values appear locally, indicating that the gravity current is detrained into the ambient fluid. The velocity profiles of gravity current develop multi-peak patterns in stratified environments due to fingering intrusive patterns. Our analysis reveals that as the vegetation density increases, the generated wakes behind vegetation cylinders increase local entrainment and mixing, causing the density of current flow from vegetation to decrease and reach the neutral buoyancy layer of ambient fluids earlier, finally leading to a smaller separation depth.
\end{abstract}

Keywords: gravity currents; vegetation patches; stratified environment; entrainment; velocity profile; separation depth

\section{Introduction}

Gravity currents, also called density currents, are driven by the density difference between two fluids, mainly in the horizontal direction [1]. They are important processes both in natural and artificial situations and can significantly affect the ecological environment of water bodies. For example, thermal wastewater from power plants causes serious problems in the aquatic environment [2]. The intrusion of saltwater into freshwater in estuary areas deteriorates water quality. Gravity currents are widely generated in lakes, estuaries, and oceans with a certain bed slope [3-8]. In these environments, vertical variations in fluid density (also called stratification) commonly exist due to the uneven temperature distribution or diffusion of salinity. In addition, vegetation patches (e.g., reeds, mangroves) in the nearshore are another environmental factor that directly influences the propagation and dynamics of gravity currents. Therefore, to understand the propagation of downslope 
gravity currents in real conditions, both stratified environments and vegetation patches are crucial environmental factors that must be considered.

Previous studies have extensively investigated the individual effects for different environmental conditions on the dynamics and propagation of downslope gravity currents. In stratified environments, downslope gravity currents could horizontally separate from the slope at the location where the current density and density of ambient fluids are equal (also called the neutral buoyancy layer); moreover, multiple intrusions (fingering) of current appear, and the separation depth decreases with the increasing stratification level of the ambient water [5,9]. These studies also revealed that the stratified environments generally reduce turbulent mixing and current head velocity [5]. In stratified environments, the descending currents have two stages: initial acceleration and subsequent deceleration according to the balance of inertial, buoyancy, and friction forces [10]. On the other hand, aquatic vegetation commonly found in nearshore regions is another environmental parameter that affects the motions of gravity currents. Nearshore vegetation could significantly reduce the propagation velocity of gravity currents, alter the morphological and turbulent structure of the gravity current head, and complicate material transport processes [11-13]. Furthermore, the dynamic characteristics of gravity currents could be affected by vegetation density, submergence, distribution, and so on $[12,14,15]$. However, few studies have comprehensively investigated the combined impact of a stratified environment and vegetation patches on the dynamic characteristics of the downslope gravity current. The authors of [16] investigated the influence of ambient stable stratification on the gravity current propagating over submerged vegetation patches and found that the dilution of the current density is enhanced by denser vegetation patches but is weakened by stronger ambient stratification. Nevertheless, their study only focused on the phenomena over a flatbed, which may not meet some practical conditions in nearshore regions. The dynamic features of gravity currents in stratified and sloping environments can be much more complicated than those in homogeneous and flat environments [5]. In particular, it is still unclear how emergent vegetation patches adjust the separation processes of gravity currents in stratified and sloping environments.

In the present study, combined experimental and numerical investigation (threedimensional large eddy simulation, 3D LES) were used to elucidate the impact of stratified environments and vegetation patches on downslope gravity currents, including the head velocity, entrainment process, separation depth, and velocity profile. The reliability of the numerical model was verified by corresponding laboratory experiments. This paper is organized as follows. Section 2 describes the experimental and numerical setup and relevant parameters. The comparison results are provided and discussed in Section 3. Finally, the main conclusions are given in Section 4. The conclusions drawn can provide a scientific basis for the propagation and separation of gravity currents in nearshore stratified environments.

\section{Descriptions of Experiments and Simulations}

\subsection{Experimental Apparatus}

Lock-exchange experiments were carried out using a similar apparatus to that of [5], as shown in Figure 1. The dimensions of the rectangular flume were $280 \mathrm{~cm}$ in length $(L)$, $15 \mathrm{~cm}$ in width $(W)$, and $46 \mathrm{~cm}$ in height $(H)$. A double-tank system with accurate flow-rate control was adopted to create linearly stratified environments, and the accuracy of the stratification level of the ambience was evaluated using a self-designed sampler to obtain water samples (refer to [5] for details). The correlation coefficient $R^{2}$ between the sampling value and the preset values of all experiments was greater than 0.98 . The lock gate divided the flume into two parts: the right part was a $19-\mathrm{cm}$-long chamber that contained the dense current (saline mixture) within the initial density $\rho_{c o}$ and height $h_{0}$, and the left part was a 261-cm-long experimental zone filled with homogeneous/stratified ambient fluid. The slope angle $\theta$ was $9^{\circ}$. In this study, emergent vegetation was mimicked using an array of vertical cylinders located in ambient water regions, which started at $38 \mathrm{~cm}$ from the gate 
(two times of the lock chamber length; see Figure 1). The cylinders were made of rigid wood with a diameter of $D=7 \mathrm{~mm}$, and the length $L_{v}$ and width $W_{v}$ of the vegetation region were 30 and $15 \mathrm{~cm}$, respectively. The cylinders were randomly distributed to better represent natural vegetation conditions [17]. The vegetation density was quantified using the solid plant fraction $(S P F)$, which was defined as a fractional area of the bed occupied by the cylinders, i.e., $S P F=N \pi(D / 2)^{2} /\left(L_{v} W_{v}\right)$, where $N$ is the number of cylinders. To visualize the flow and capture the details of the propagation process, the gravity current was dyed with appropriate potassium permanganate. The salinity of the gravity current was measured by a 79017 ATAGO salt meter with an accuracy of 0.1 parts per thousand, and then corresponding densities were calculated using the seawater state equation $[17,18]$. All experiments were carried out at constant room temperature $\left(25^{\circ} \mathrm{C}\right)$ to eliminate the density variation due to temperature differences. The overall flow motions and evolution patterns of gravity currents were recorded using a charge-coupled device (CCD) camera with $1920 \times 1080$ pixels of resolution and an acquisition frequency of $25 \mathrm{~Hz}$. A calibration board was positioned in the flume for horizontal and vertical geometric calibration. By rapidly lifting the gate, gravity currents were generated down the slope.

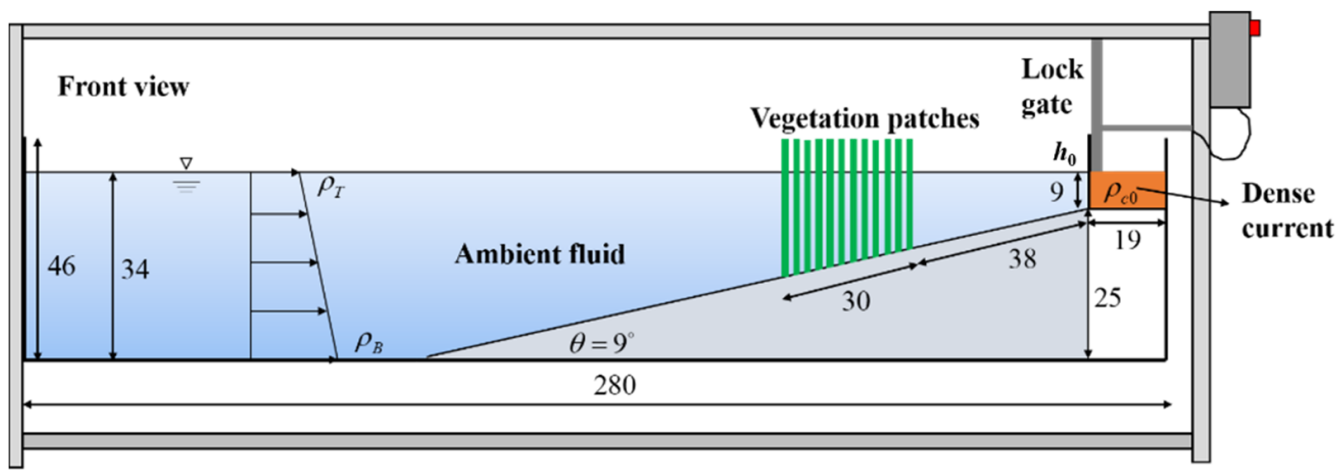

Plan view

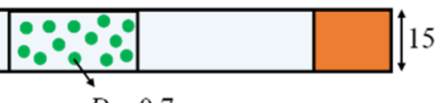

$D=0.7$

Figure 1. The schematic of the experimental setup (unit: $\mathrm{cm}$ ).

\subsection{Numerical Setup}

In this study, the LES-filtered Navier-Stokes equations for time-varied, three-dimensional, and incompressible stratified flows were adopted, as given in tensor notation by

$$
\frac{\partial\left(\rho u_{i}\right)}{\partial t}+\frac{\partial\left(\rho u_{i} u_{j}\right)}{\partial x_{j}}=-\frac{\partial p}{\partial x_{i}}+\mu \frac{\partial^{2}\left(u_{i}\right)}{\partial x_{j} \partial x_{j}}-\frac{\partial\left(\rho \tau_{i j}^{S G S}\right)}{\partial x_{j}}-\rho g_{i}
$$

with the filtered continuity equation

$$
\frac{\partial u_{i}}{\partial x_{i}}=0
$$

and the filtered density transport equation

$$
\frac{\partial \rho}{\partial t}+\frac{\partial\left(\rho u_{j}\right)}{\partial x_{j}}=\kappa \frac{\partial^{2} \rho}{\partial x_{j} \partial x_{j}}-\frac{\partial \chi_{j}^{S G S}}{\partial x_{j}}
$$

where $u_{i}$ is the Cartesian components of the filtered velocity field, where the index $i=1,2$, and 3 indicates the $x$ (streamwise), $y$ (spanwise), and $z$ (vertical) directions, respectively; $p$ is the filtered pressure, $\rho$ is the filtered local density, $g$ is the gravitational acceleration, $\mu$ is the dynamic viscosity, $\kappa$ is the molecular diffusivity of the saline leading to the density 
variation, and $t$ is time. The closure of the subgrid-scale (SGS) stress tensor $\tau_{i j}^{S G S}$ and the subgrid-scale scalar flux vector $\chi_{k, j}^{S G S}$ in Equations (1) and (3) are solved using the WALE model [19]. To model motions of gravity currents, the multiphase-flow modeling approach based on mixture theory was adopted [19]. The mixture model treats all the phases included in the simulations as interpenetrating continua. The details of the mixture models are given in [20]. In the study, Equations (1)-(3) were solved using the combined mixture model with LES-type turbulence models in the computational fluid dynamics software ANSYS Fluent.

The reference water phase $\left(\rho_{w}=998.2 \mathrm{~kg} / \mathrm{m}^{3}\right)$ was introduced in the multiphase-flow model and combined with the Custom Field Function in the ANSYS Fluent software to realize the density stratified environment, i.e., the linear transition from the larger density at the bed $\rho_{B}$ to the smaller ones at the surface $\rho_{T}$. Plenty of individual cylinders with $D=7 \mathrm{~mm}$ were placed in the computational domain to simulate the vegetation patches starting at $38 \mathrm{~cm}$ from the top of the slope as the experimental setup. Two vegetation arrangements, i.e., in-line and staggered configurations, were considered and compared to each other and the experimental results. For boundary conditions, the sides and bottom of the computational domain were set as no-slip wall, and the top of the domain was a no-shear stress condition, i.e., zero velocity gradient.

The Semi-Implicit Method for Pressure Linked Equations (SIMPLE) scheme that especially fits the complex flow and boundary conditions was adopted as the time marching method to ensure convergence and stability [21,22]. For the spatial discretization, the gradient term used the Least Square Cell-Based method, the pressure term employed the algorithm PRESTO!, and the volume fraction term adopted the Central Difference Scheme, which can obtain more accurate results in the LES turbulence model $[12,16]$. The under-relaxation factors of the pressure, density, and momentum were set as $0.3,1$, and 0.7 , respectively. For the calculation setting, the time step size equal to $0.01 \mathrm{~s}$ was chosen to ensure the model's stability and convergence. The residuals were less than $10^{-4}$ when the calculation was converged in each time step.

The Solidworks software was used to establish the computational domain similar to the experiment flume, as shown in Figure 1. The unstructured tetrahedral mesh was made in the fluid domain and the meshes near vegetation cylinders were refined, as shown in Figure 2. The ratio range of the mesh size adjacent to the cylinder to the diameter of the cylinder $D$ was $1 / 70 \sim 1 / 7$. The detailed configuration of the numerical meshes is presented in Table 1.

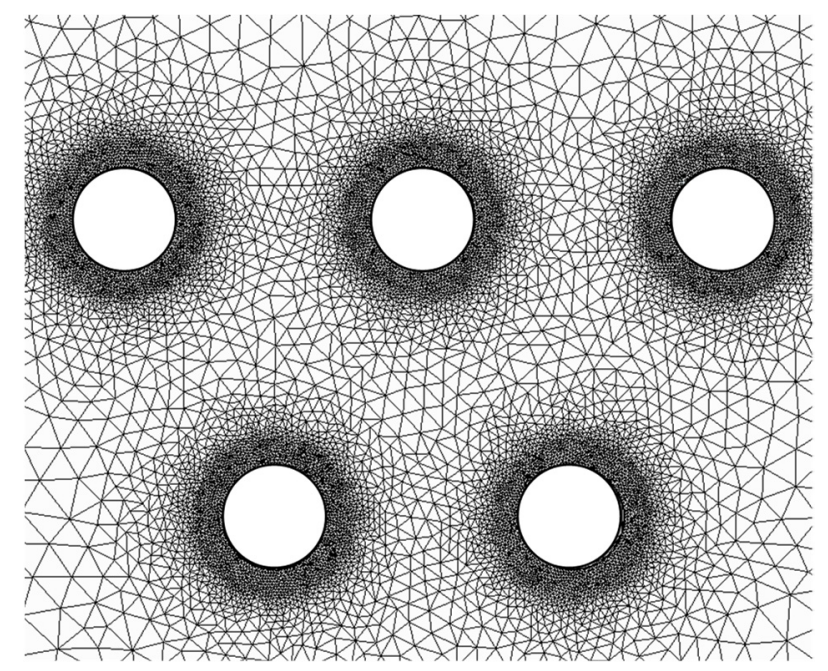

Figure 2. A zoom-in view of the meshes around vegetation cylinders for staggered configurations. 


\subsection{Study Parameters}

In this study, five laboratory experiments and seven numerical simulations were conducted, and the corresponding parameters are listed in Table 1 . The relative magnitude of the stratification $S$ is defined as [18]:

$$
S=\frac{\rho_{B}-\rho_{T}}{\rho_{c 0}-\rho_{T}}
$$

where $\rho_{B}$ and $\rho_{T}$ are the fluid density at the bottom and top of the ambience, respectively. The relative magnitude of the stratification $S$ is set larger than one so that the gravity current would separate from the slope [5].

Table 1. Parameters of the experimental and numerical runs.

\begin{tabular}{|c|c|c|c|c|c|c|c|c|c|}
\hline \multirow[b]{2}{*}{ Runs } & \multicolumn{6}{|c|}{ All Applicable Parameters } & \multicolumn{3}{|c|}{ Parameters Only Describing Simulations } \\
\hline & $L_{v}(\mathrm{~cm})$ & $H_{v}(\mathrm{~cm})$ & $S P F(\%)$ & $S$ & $\underset{\left(\mathrm{cm} / \mathrm{s}^{2}\right)}{g^{\prime}}$ & $\begin{array}{l}\text { Vegetation } \\
\text { Arrangement }\end{array}$ & $\begin{array}{c}\text { Total Number } \\
\text { of Mesh }\end{array}$ & $\begin{array}{l}\text { Average } \\
\text { Mesh Size } \\
\text { (mm) }\end{array}$ & $\begin{array}{c}\text { Range of the Mesh } \\
\text { Size in the } \\
\text { Vegetated Zone } \\
\text { (mm) }\end{array}$ \\
\hline ES-N0 ${ }^{1}$ & 0 & 0 & 0 & 0 & 11.85 & N.A. & $2,801,142$ & 3 & N.A. \\
\hline ES-S0 & 0 & 0 & 0 & 1.1 & 11.85 & N.A & $2,807,569$ & 3 & N.A. \\
\hline S-S1 & 30 & 30 & 0.5 & 1.1 & 11.85 & I & $5,814,890$ & 4 & $2 \sim 0.1$ \\
\hline ES-S2 & 30 & 30 & 4.5 & 1.1 & 11.85 & I & $5,896,949$ & 4 & $2 \sim 0.1$ \\
\hline ES-S3 & 30 & 30 & 9 & 1.1 & 11.85 & I & $5,986,091$ & 4 & $2 \sim 0.1$ \\
\hline ES-S4 & 30 & 30 & 18 & 1.1 & 11.85 & $\mathrm{I}, \mathrm{S}^{2}$ & $6,078,224$ & 4 & $2 \sim 0.1$ \\
\hline S-S5 & 30 & 30 & 30 & 1.1 & 11.85 & $\mathrm{I}$ & $6,095,917$ & 4 & $2 \sim 0.1$ \\
\hline
\end{tabular}

1 "ES" represents the runs carried out by experiments and simulations, and "S" denotes the runs only done with simulations. " "I" denotes in-line vegetation arrangements, and " $\mathrm{S}$ " represents staggered vegetation arrangements.

The driving force for gravity current is the reduced gravity $g^{\prime}$, which is given by

$$
g^{\prime}=\frac{\rho_{c 0}-\rho_{T}}{\rho_{c 0}} g,
$$

where $g\left(=9.81 \mathrm{~m} / \mathrm{s}^{2}\right)$ is the gravitational acceleration. The front location of the current head $x_{f}$ is defined as the along-slope distance of the foremost of gravity current from the lock gate, and the corresponding current velocity is represented as: $u_{f}=d x_{f} / d t$. To non-dimensionalize the experimental parameters, the following characteristic parameters were used, i.e., characteristic length $h_{0}$, characteristic velocity $\left(g^{\prime} h_{0}\right)^{1 / 2}$, and characteristic time $h_{0} /\left(g^{\prime} h_{0}\right)^{1 / 2}$, as the references [5,6] did. The non-dimensionalized location of the current head $X_{f}$, current velocity $U_{f}$, and propagation time $T$ are expressed as:

$$
\begin{gathered}
X_{f}=\frac{x_{f}}{h_{0}}, \\
U_{f}=\frac{u_{f}}{\left(g^{\prime} h_{0}\right)^{1 / 2}}, \\
T=\frac{t}{h_{0} /\left(g^{\prime} h_{0}\right)^{1 / 2}} .
\end{gathered}
$$

\section{Results and Discussion}

\subsection{Mesh Independence Study and Comparison of Experimental and Numerical Results}

To determine the mesh resolution for the simulations, the mesh-size independence study was first performed. Runs ES-S0 and ES-S3 were selected with three levels of mesh sizes: fine, medium, and coarse. Corresponding parameters such as the total number of meshes, average mesh size, and average mesh size in the vegetated zone are provided in Table 2. The non-dimensional front location of the gravity current $X_{f}$ was used to check the 
mesh independence and validate the numerical model in comparison with experimental measurements. From the laboratory measurements, the current forefront can be easily identified by the color contrast between the current and ambient fluids. On the other hand, in the numerical simulations, the current forefront was determined by a threshold phase concentration value $c$ of 0.01 , suggested by [6]. They also found that the threshold phase concentration ranging between 0.01 and 0.1 has negligible effects on $X_{f}$.

Table 2. Parameters of numerical runs for the mesh independence study.

\begin{tabular}{ccccc}
\hline Runs & Mesh Level & $\begin{array}{c}\text { Total Number } \\
\text { of Meshes }\end{array}$ & $\begin{array}{c}\text { Average Mesh } \\
\text { Size } \mathbf{( m m )}\end{array}$ & $\begin{array}{c}\text { Average Mesh Size in the } \\
\text { Vegetated Zone } \mathbf{( m m})\end{array}$ \\
\hline ES-S0 & Coarse & $1,200,845$ & 4 & N.A. \\
ES-S0 & Medium & $2,807,569$ & 3 & N.A. \\
ES-S0 & Fine & $5,031,284$ & 2 & N.A. \\
ES-S3 & Coarse & $1,043,647$ & 7 & 2 \\
ES-S3 & Medium & $5,986,091$ & 4 & 1 \\
ES-S3 & Fine & $8,552,419$ & 3 & 0.5 \\
\hline
\end{tabular}

Figure 3 shows the comparisons of $X_{f}-T$ between numerical runs with different mesh levels and the corresponding experimental measurements. For the bare sloping bed (Figure 3a,b, Runs ES-N0 and ES-S0), the non-dimensional front locations of the current head for medium and fine mesh were close to each other, while the case of the coarse mesh deviated from them. However, for a sloping bed with emergent and rigid vegetation patches (Figure 3c, Run ES-S3), the $X_{f}-T$ relation values for three grid levels were all close to each other. Considering the accuracy of the results and the time-consuming calculation, the medium mesh size was chosen to simulate downslope gravity currents through rigid vegetation patches. After comparing the simulations (medium mesh) with the corresponding experimental measurements, the average relative errors $\left(\left|\frac{X_{f, \text { simulation }}-X_{f, \text { experiment }}}{X_{f, \text { simulation }}}\right| \times 100 \%\right)$ of the whole propagation process for Runs ES-N0, ES-S0, and ES-S3 were found to be $1.23 \%, 1.19 \%$, and $6.47 \%$, respectively, suggesting that the numerical model with the present medium mesh can accurately simulate the motions of gravity currents with and without emergent and rigid vegetation patches.

In addition, different vegetation arrangements on the movements of gravity current were also compared. Figure 4 presents the comparisons of $X_{f}-T$ between the vegetation patches with the in-line and staggered arrangements. It is found that they are very close (average relative error of the whole current propagation process is approximately 1.07\%), so the in-line arrangement was adopted in this study to analyze the effect of emergent vegetation on the downslope gravity current.

\subsection{Morphological Patterns of Gravity Currents}

Figure 5 shows the three-dimensional morphological patterns of gravity currents in homogeneous and stratified environments. In homogeneous environments (Figure 5a), as the gravity currents move down the slope, the Kelvin-Helmholtz $(\mathrm{K}-\mathrm{H})$ billows (caused by the velocity shear instability) occur at the interface between the current and ambient water, as previous studies observed [5,23]. The K-H instability promotes fluid mixing and entrainment at the interface of the current and ambient water. On the other hand, in stratified environments (Figure 5b), since the driving forces, i.e., the density differences causing the reduced gravity $g^{\prime}$, become weaker as the current propagates into deep regions, the current will move slowly and finally separate from the slope and intrude horizontally into the locations where the densities of the current and ambient fluids are equal (neutral buoyancy layer). In addition, the volume fraction of the gravity current along the slope shows that the current head is the core area to maintain the driving buoyancy, and the density at the current wake decreases gradually with the propagation processes. 

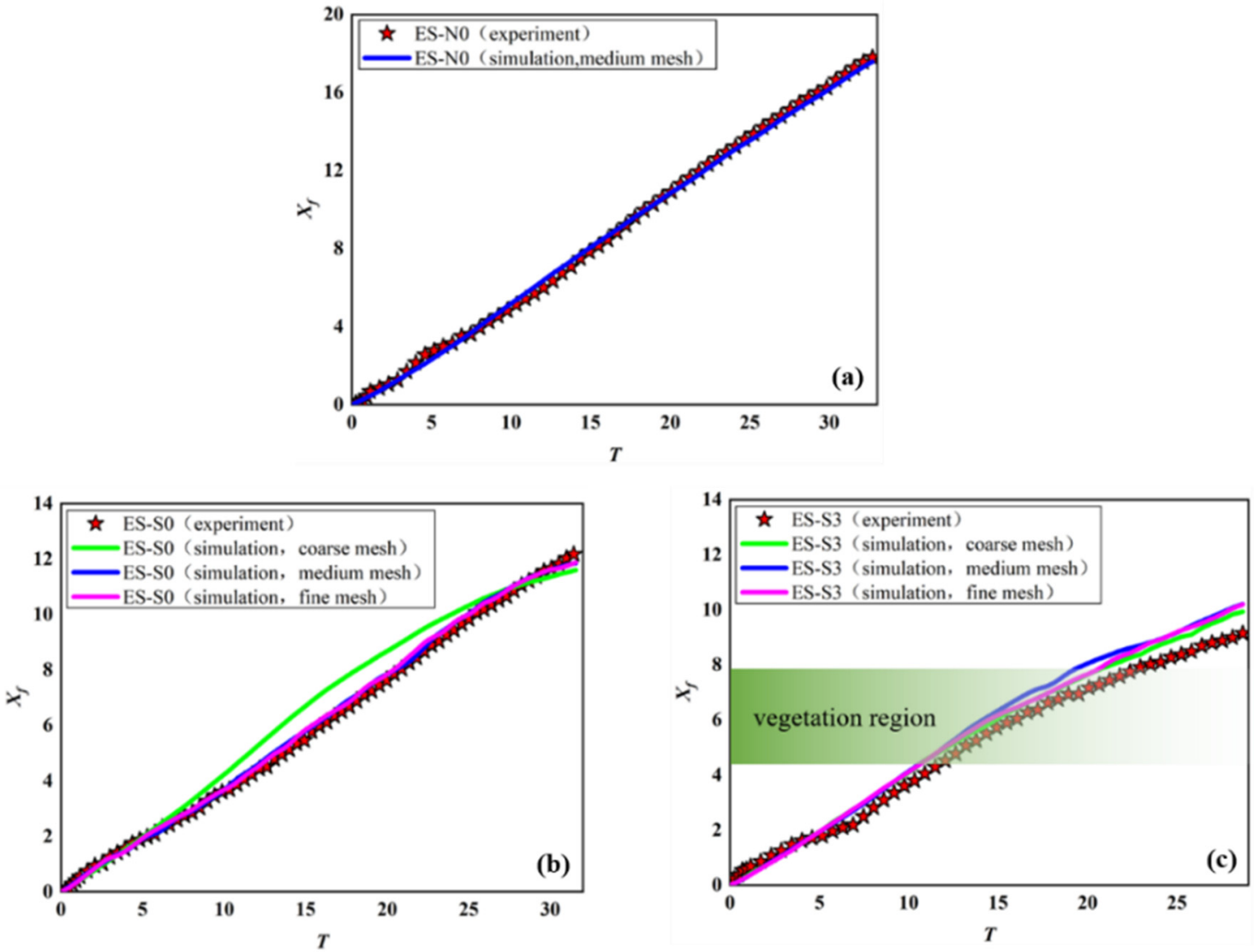

Figure 3. Comparisons between simulations with different mesh levels and the corresponding experimental measurements. (a) ES-N0, (b) ES-S0, and (c) ES-S3 (green rectangle denotes the vegetation region).

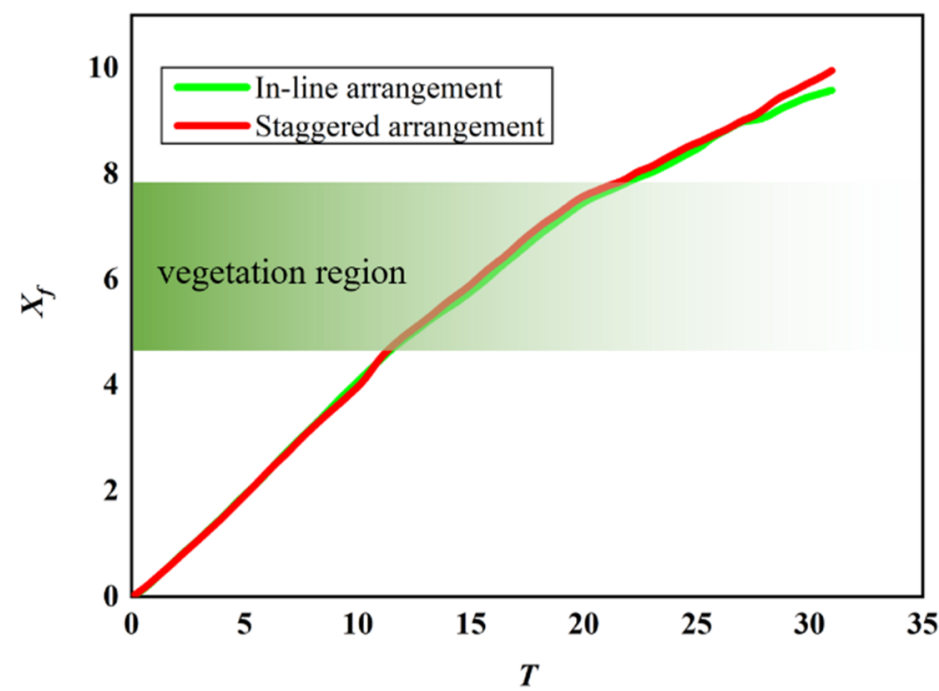

Figure 4. Comparisons of $X_{f}-T$ between vegetation patches with the in-line arrangement and staggered arrangement (Run ES-S4). The green rectangle represents the vegetation region. 
(a1) $t=8 \mathrm{~s}$

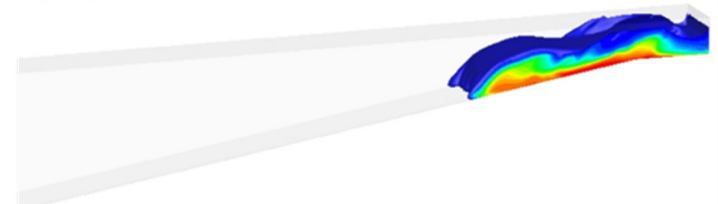

(a2) $t=12.5 \mathrm{~s}$

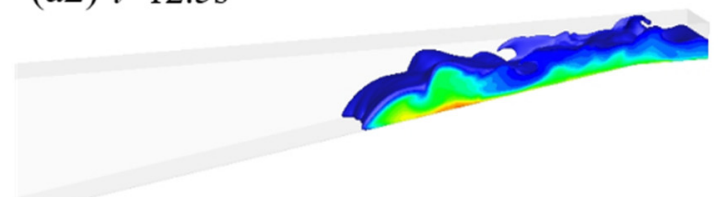

(a3) $t=22 \mathrm{~s}$

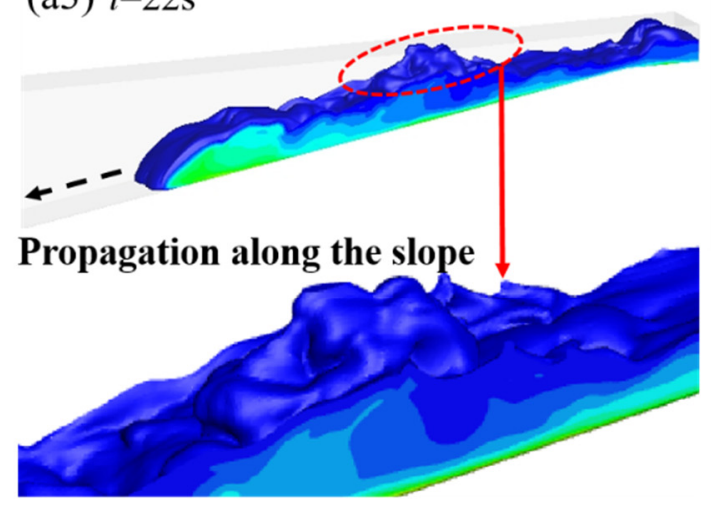

(b1) $t=8 \mathrm{~s}$

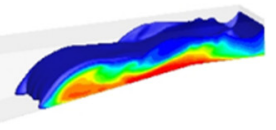

(b2) $t=14 \mathrm{~s}$

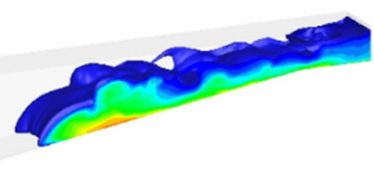

(b3) $t=29 \mathrm{~s}$

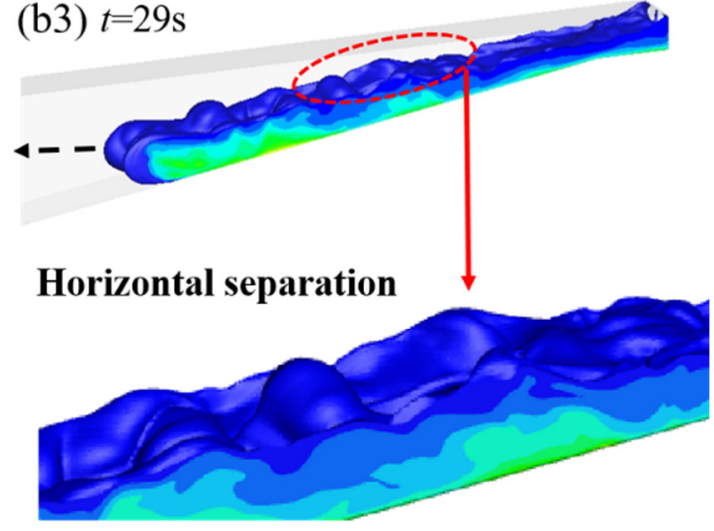

(b)

(a)

volume fraction

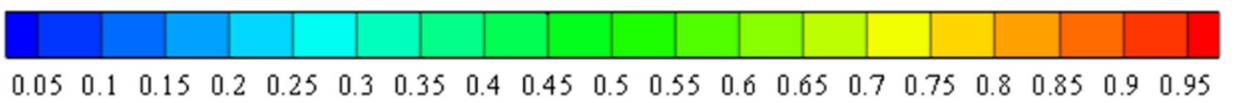

Figure 5. The three-dimensional simulation results of gravity current flowing along the slope in the homogeneous and stratified environments: (a) Run ES-N0; (b) Run ES-S0.

In stratified environments, before the gravity currents propagate into vegetation patches (Figure 6(a1)), the morphological patterns of gravity currents are approximately the same as the run without vegetation (Run ES-S0, Figure 5(b1)), indicating that the presence of vegetation patches will not affect the upstream flow patterns. After currents flow into vegetation patches (Figure 6(b1)), vegetation drag suppresses current mixing and entrainment, i.e., the K-H instability, with ambient fluids, and the current head transforms from a classic semi-elliptic shape (inertia-dominant) to a triangular shape (drag-dominant), as [15] revealed. Some dense fluids are blocked in front of vegetation patches and mixed with local ambient fluid, leading to the locally elevated K-H instability at the interface of current-ambient fluid in the region (Figure 6(b2)). Furthermore, the presence of vegetation cylinders also leads to severe lateral non-uniformity of the current front, causing more evident lobes and clefts of the current head structure, as shown in Figure 6(b2).

\subsection{Front Velocity}

In homogeneous environments, the propagation processes of typical downslope gravity currents can be divided into an acceleration phase and a constant phase [4]. For the stratified environments, a separation phase will exist when the relative magnitude of stratification $S>1$ [6]. The presence of vegetation patches complicates the evolutionary process of gravity currents [8]. Figure 7 provides the lateral-averaged front velocity of 
the gravity current head with different emergent vegetation densities. For experimental runs ES-S0 to S-S5 in stratified environments, the $U_{f}-X_{f}$ curves were obtained before the flow separating from the slope. From Figure 7, it can be seen that, at the beginning, i.e., the release of dense fluids from the gate, the gravity currents for all numerical runs follow a similar trend, i.e., the currents experience sudden acceleration. For Run ES-N0 in a homogeneous environment, the current first accelerates, reaches peak velocity $\left(U_{f} \approx 0.54\right)$, and then propagates at an approximately constant velocity, as [4] reported. For Run ES-S0 in a stratified environment (without vegetation), the smaller driving force leads to less peak velocity $\left(U_{f} \approx 0.43\right)$ in comparison with Run ES-N0 in a homogeneous environment. Furthermore, the decreasing driving force also gradually reduces the current velocity for Run ES-S0 until the current separates from the slope.

(a1) $t=9 \mathrm{~s}$

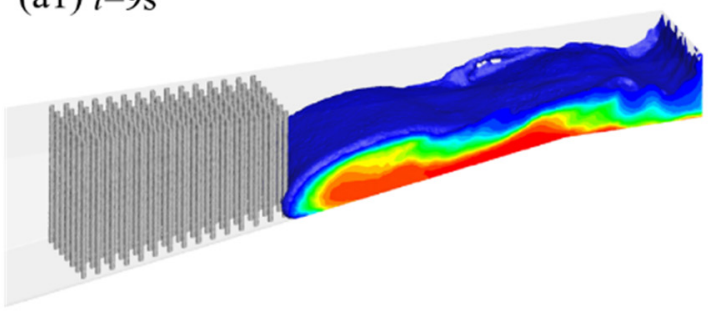

(a2)

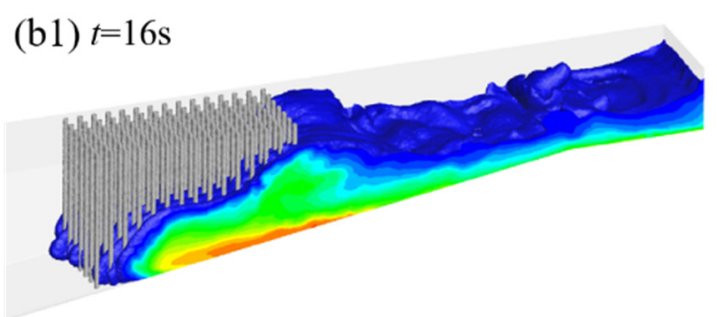

(b2)

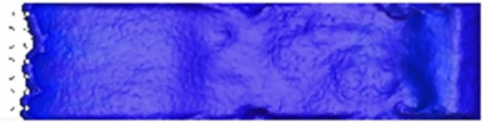

(a)

volume fraction

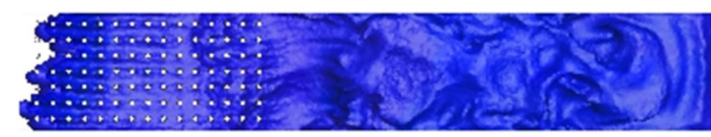

(b)

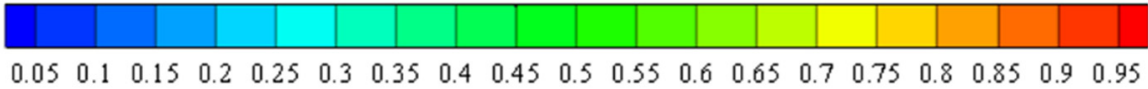

Figure 6. The three-dimensional simulation results of gravity current flowing through emergent vegetation patches along the slope in the stratified environment (Run ES-S3): (a) before vegetation patches $(t=9 \mathrm{~s})$, and $(\mathbf{b})$ within vegetation patches $(t=16 \mathrm{~s})$. Note: $(\mathbf{a} 1, \mathbf{b} \mathbf{1})$ are the three-dimensional view of the flow, and $(\mathbf{a} 2, \mathbf{b} 2)$ are the top view of the flow.

The influence of rigid vegetation patches on the propagation processes of the downslope gravity current in stratified environments can be summarized into three perspectives. First, before the current encounters vegetation patches, the flow patterns of gravity currents are almost the same for all simulations, meaning that the vegetation impact is insignificant at this propagation stage. Second, when gravity currents flow through vegetation in stratified environments, the current velocity is greatly reduced, and the level of velocity reduction is proportional to vegetation density due to greater vegetation drag. Finally, the transition point of current velocity, i.e., from acceleration to deceleration phases, appears earlier as the vegetation becomes denser. This is because of the changes in force balance among the driving force, inertia force, and vegetation drag, which is also reflected in the morphology of the current head, as mentioned above. For the dense vegetation Runs ES-S4 and S-S5, the phase transformation is even advanced to the beginning of the vegetation region, and the front velocity of the current head after passing through the vegetation patches is attenuated by around 40\% (relative to Run ES-S0, in stratified environment and without vegetation). 


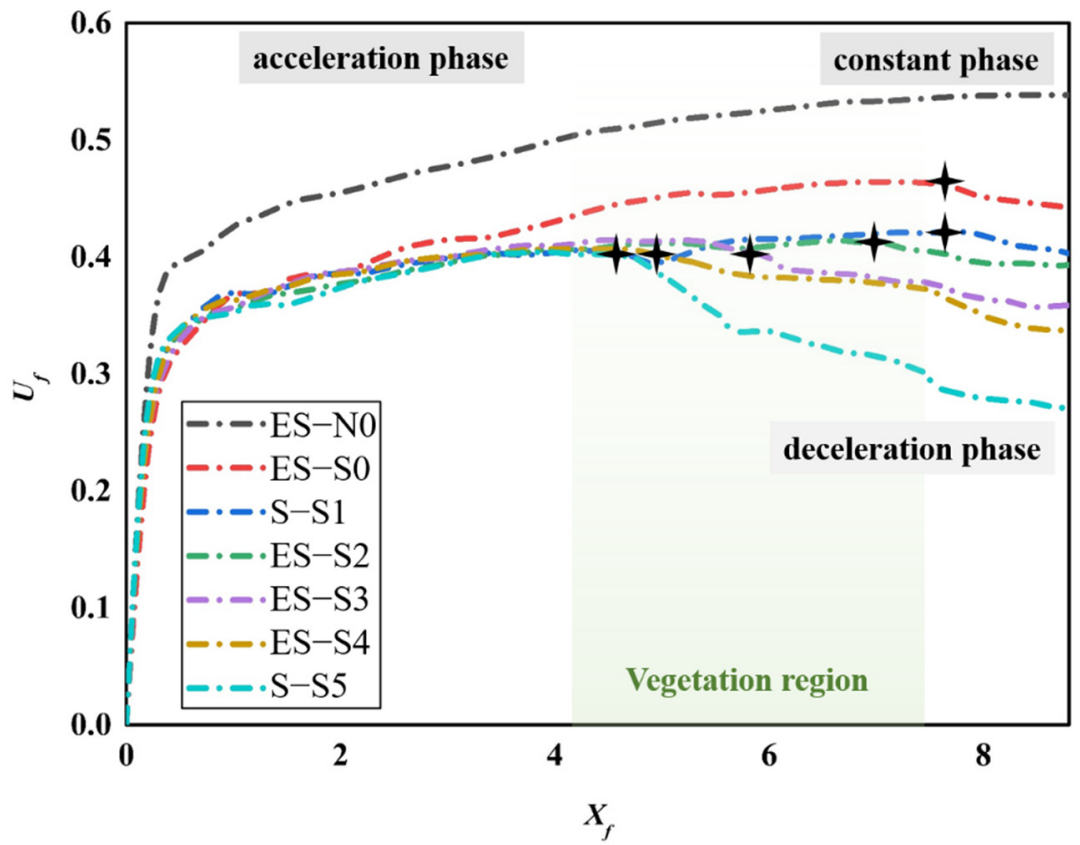

Figure 7. Spatial evolution of the dimensionless front velocity of gravity current. The light green rectangle marks the vegetation region, and the black asterisk indicates the transition point between the acceleration and deceleration phases.

\subsection{Entrainment Process}

The entrainment process is crucial to determine the evolution and extinction of the gravity current. The bulk entrainment coefficient $E_{\text {buik }}$ and instantaneous entrainment coefficient $E_{\text {inst }}$ at a certain time $t_{i}$ can be defined as [6]:

$$
\begin{gathered}
E_{\text {bulk }, i}=\frac{W_{\text {bulk }, i}}{u_{f, i}} \\
E_{\text {inst }, i}=\frac{W_{\text {inst }, i}}{0.5\left(u_{f, i}+u_{f, i-1}\right)} \\
u_{f, i}=\frac{x_{f, i}}{t_{i}}
\end{gathered}
$$

where the subscript $i$ refers to the time step, and $W_{b u l k, i}$ and $W_{\text {inst, } i}$ are the bulk and instantaneous water entrainment velocities, respectively, which can be calculated by

$$
\begin{gathered}
W_{b u l k, i}=\frac{Q_{b u l k, i}}{S_{i}} \\
W_{\text {inst }, i}=\frac{Q_{\text {inst }, i}}{0.5\left(S_{i}+S_{i-1}\right)}
\end{gathered}
$$

where $S_{i}$ is the length of the upper interface of the current, and $Q_{b u l k, i}$ and $Q_{i n s t, i}$ are the effective bulk and instantaneous water discharges from the ambient environment to the gravity current, respectively. Since the upper interface between the current and ambient water varies with respect to time, we used the first-order relationship proposed by [6,24], i.e., $S_{i}=\left(h_{0}+l_{0}+x_{f, i}\right) \times W$, to calculate $S_{i}$. According to reference [6], the terms $Q_{b u l k, i}$ and $Q_{i n s t, i}$ in Equations (12) and (13) can be expressed as $Q_{b u l k, i}=\left(V_{i}-V_{0}\right) / t_{i}$ and $Q_{\text {inst }, i}=\left(V_{i}-V_{i-1}\right) /\left(t_{i}-t_{i-1}\right)$, respectively. $V_{i}$ is the current volume at the time $t_{i}$ and $V_{0}$ is the initial current volume in the chamber. In the three-dimensional computational domain, the mesh with local concentration $c$ greater than 0.01 was identified and its volume was summed to obtain the corresponding volume of the gravity current. 
Figure 8 depicts the spatial variations in the three-dimensional bulk entrainment coefficient $E_{\text {buik }}$ and instantaneous entrainment coefficient $E_{\text {inst }}$. On the whole, at the initial propagation of the gravity current, due to the gravitational collapse, a large amount of ambient fluid is entrained and $E_{b u l k}$ reaches the peak value instantly (Figure 8a). In stratified environments (Run ES-S0), initial $E_{b u i k}$ values are larger than the counterpart ones in homogeneous environments (Run ES-N0). This is because gravity currents in stratified environments tend to horizontally intrude into the neutral buoyancy layer at the beginning stage and enlarge the mixing regions with ambient fluids, which is favorable to entrainment processes. As the current propagates, the stratification inhibits turbulent generation as well as fluid entrainment, causing $E_{\text {buik }}$ values in homogeneous and stratified environments to be close to each other. For the simulations with the vegetation patches (Runs ES-S1-5), it can be found that the peak value of $E_{b u i k}$ at the initial current propagation is inversely proportional to the vegetation density. This is attributed to the emergent and rigid vegetation blocking the convective channel of the upper ambient fluid to significantly inhibit the shear instability process at the interface of the gravity current. Thus, the entrainment process is weakened. After gravity currents flow through the vegetation region, $E_{\text {buik }}$ for vegetation runs (Runs ES-S1-5) is larger than the one without vegetation (Runs ES-N0 and ES-S0). As the vegetation becomes denser, the $E_{\text {buik }}$ values will converge to greater values. The phenomena can be attributed to two aspects: on the one hand, the wake generated by vegetation stems enhances fluid entrainment and mixing; on the other hand, the gravity current in dense vegetation would separate earlier from the slope (see next section), also enlarging the contact volume as well as entrainment between the current and ambient fluids.
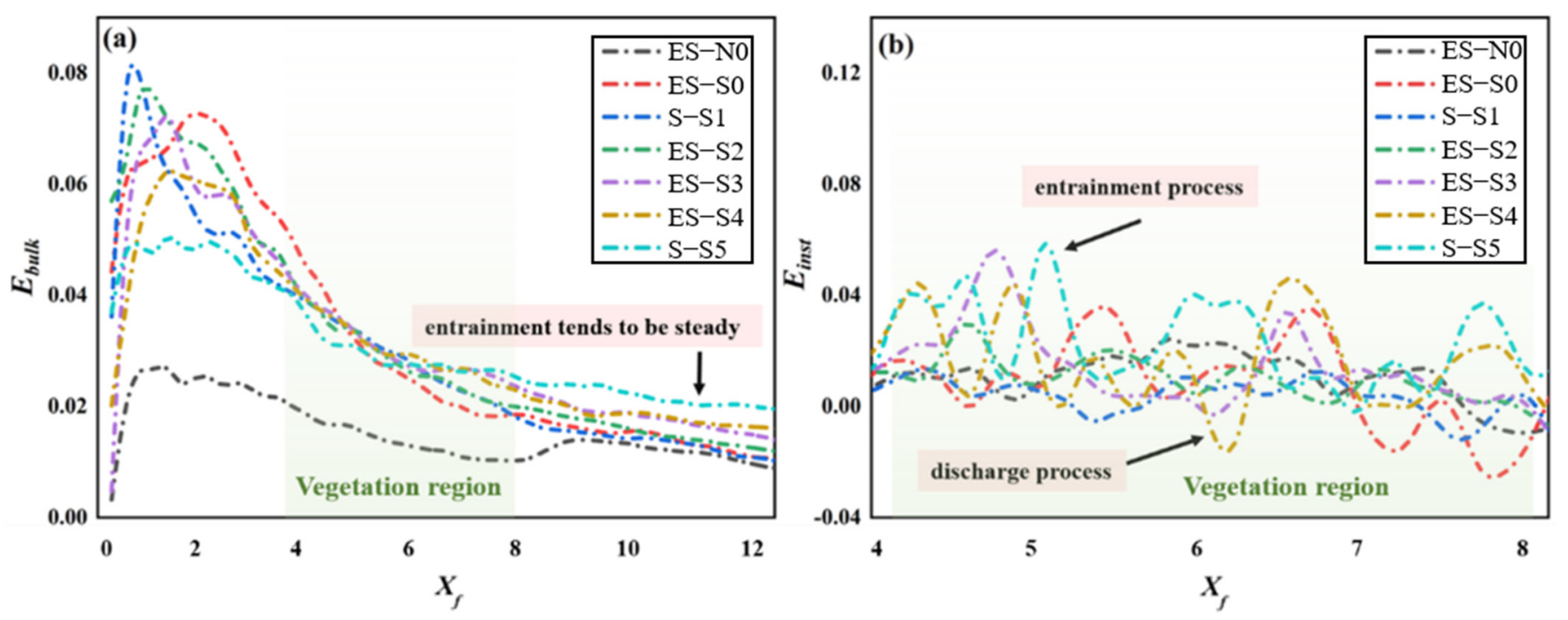

Figure 8. Spatial evolution of the three-dimensional bulk entrainment coefficient $E_{b u i k}$ (a) or instantaneous entrainment coefficient $E_{\text {inst }}(\mathbf{b})$. The light green rectangle marks the vegetation region.

Compared with the trend of $E_{\text {buik }}$ along the current propagation, $E_{\text {inst }}$ fluctuates severely (as shown in Figure 8b), suggesting local, intense turbulent mixing and entrainment. In addition, the emergent and rigid vegetation patches make the degree of fluctuation of the $E_{\text {inst }}$ more intense, and even negative values appear locally, implying that the gravity current is reversely entrained into the ambient fluid (i.e., discharge/detrainment process). Although the discharge/detrainment process will not affect the overall entrainment processes ( $E_{\text {buik }}$ is still larger than 0.01$)$, the presence of vegetation makes the vortex structures at the current interface more complex. 


\subsection{Velocity Profiles}

Velocity profiles of gravity currents can be usually assumed in the longitudinal direction ( $x$-direction). The typical velocity profile is bounded by the maximum velocity $u_{m}$ and divides into the wall and jet regions [25]. In the wall region, the gravity current is mainly affected by bottom friction, and the horizontal velocity profiles follow a power relationship [25] as

$$
\frac{u_{(z)}}{u_{m}}=\left(\frac{z}{z_{m}}\right)^{\frac{1}{n}}
$$

where $u_{m}$ is the maximum velocity of the velocity profile, $z_{m}$ is the height of the maximum velocity, and $n$ is an empirical parameter. In the jet region, the velocity profile is determined by a near-Gaussian relationship [25] as

$$
\frac{u_{(z)}}{u_{m}}=\exp \left[-\beta\left(\frac{z-z_{m}}{H_{d}-z_{m}}\right)^{\gamma}\right],
$$

where $\beta$ and $\gamma$ are empirical parameters, and $H_{d}$ is the depth-averaged current height, defined as

$$
H_{d}=\frac{\left(\int_{0}^{\infty} u d z\right)^{2}}{\int_{0}^{\infty} u^{2} d z} .
$$

Figure 9 shows the velocity profiles in the thickest part of the gravity current head in homogeneous (Run ES-N0) and stratified environments (Run ES-S0) at $t=8 \mathrm{~s}$ and $16 \mathrm{~s}$. The velocity profiles were averaged in $0.5 \mathrm{~s}$ and across the entire cross-section. At the initial stage ( $t=8 \mathrm{~s}$, Figure 9a), classical wall and jet regions are observed for both runs. Based upon mass conservation, the velocity direction in ambient fluids is opposite to the velocity direction of gravity currents (Figure 9a). Furthermore, at this stage, the dynamic characteristics of the gravity current are insensitive to environmental stratification, and the velocity profiles in homogeneous environments are slightly different from those in stratified environments, where the velocity peak in stratified environments (Run ES-S0) occurs further away from the sloping bed than the one in uniform environments (Run ES-N0). As currents propagate down the slope, the disparity between velocity profiles in homogeneous and stratified environments becomes evident, as shown in Figure 9b. In homogeneous environments, due to bed resistance, the peak velocity at $t=16 \mathrm{~s}$ reduces from 0.10 to $0.075 \mathrm{~m} / \mathrm{s}$ and the location where peak velocity appears is further away from the bed than that at $t=8 \mathrm{~s}$. According to mass conservation, the smaller current velocity in homogeneous environments enlarges the current thickness. On the other hand, for stratified environments, velocity profiles show obvious variations and display multi-peak patterns, as illustrated in Figure 9b. As mentioned above, this is because of the driving force, i.e., the density difference between the current body and ambient fluid, which gradually reduces, and the currents tend to intrude into its neutral buoyancy layer and form some fingering intrusive patterns, which induces the change in the velocity profile.

To understand the effects of vegetation patches, the velocity profile in the thickest part of the gravity current head (approximately $5 \mathrm{~cm}$ behind the current front) when the current front is at $8 \mathrm{~cm}$ before the vegetation region, $5 \mathrm{~cm}$ after the middle of the vegetation region, and $7 \mathrm{~cm}$ after the vegetation region were selected to compare the differences, as provided in Figure 10. Before vegetation patches (Figure 10a), vegetation blockage leads to strong local circulations and slightly reduces the current velocity, still satisfying the single peak pattern. Inside the vegetation region (Figure 10b), under the coupling effect of vegetation stems and the stratification environment, the typical velocity profile can be seen to be seriously distorted. When vegetation is denser, velocity becomes smaller and more uniform along the vertical direction, as in the case of an open channel flow [26]. After the vegetation region (Figure 10c), the velocity profiles are significantly changed, and the reduced level of velocity is proportional to the density of vegetation patches. In 
addition, owing to some dense fluid trapped within the vegetation patches, the current thickness dramatically decreases, especially for Run S-S5. The thickness reduces by 70\% in comparison with Run ES-N0, and part of the dense fluid is even unable to flow out from the vegetation patches.
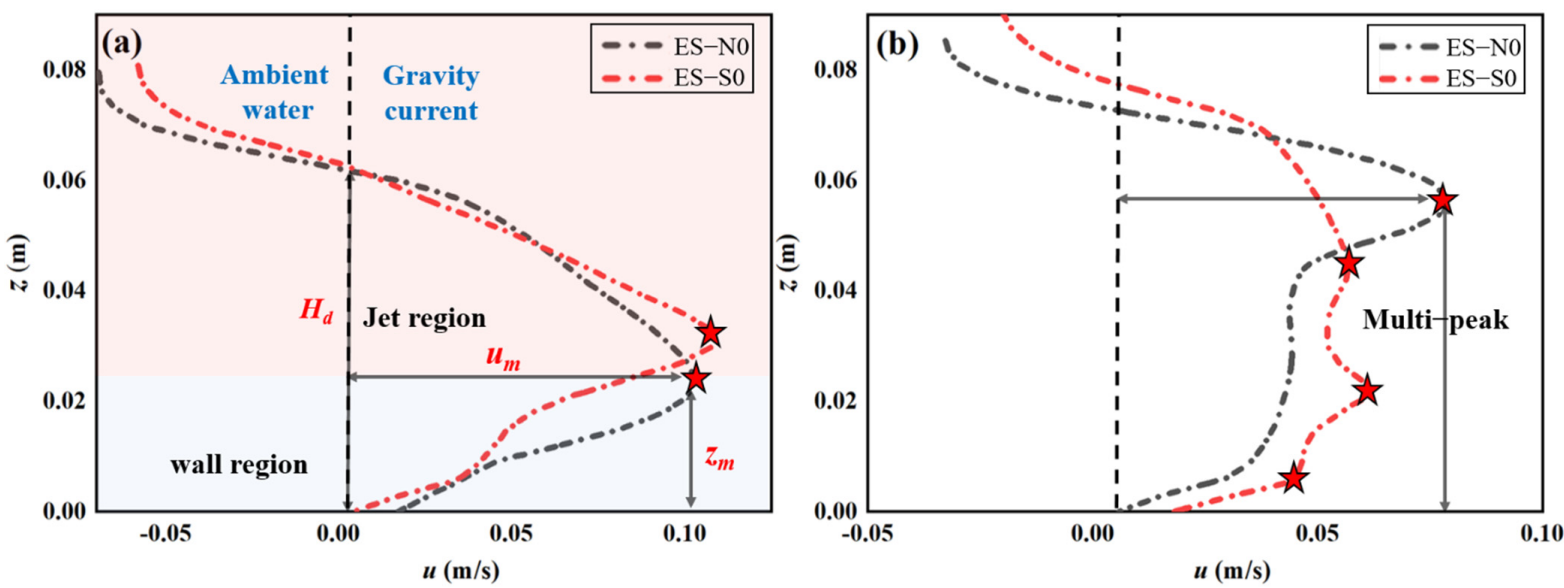

Figure 9. Comparison of the lateral averaged velocity profile in the thickest part of gravity current head in the homogeneous (Run ES-N0) and stratified environments (Run ES-S0), (a) $t=8 \mathrm{~s} ;$ (b) $t=16 \mathrm{~s}$. The vertical black dashed line partitions the gravity current from the ambient fluid according to the positive/negative values of $u$. The red asterisks represent the local maximum of the velocity profile.
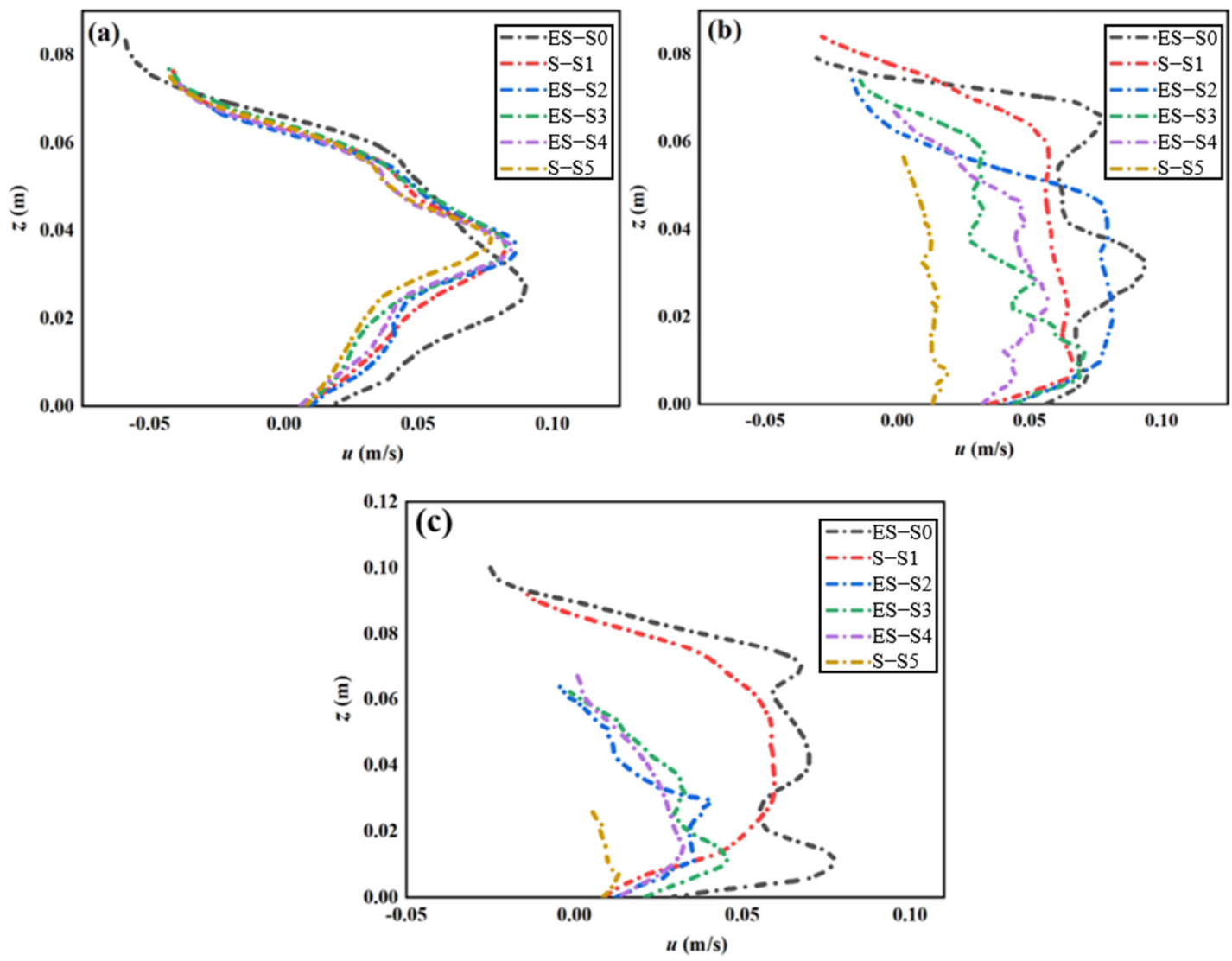

Figure 10. Comparison of the lateral averaged velocity profile in the thickest part of gravity current head (approximately $5 \mathrm{~cm}$ behind the current front) in the stratified environment: (a) the current front is at $8 \mathrm{~cm}$ before the vegetation region; (b) the current front is at $5 \mathrm{~cm}$ after the middle of the vegetation region; (c) the current front is at $7 \mathrm{~cm}$ after the vegetation region. 


\subsection{Separation Depth}

In stratified environments, during current propagation processes, the mixing and entrainment between current and ambient fluid decrease the current density, and the current finally separates from the slope at the neutral buoyancy layer, forming the intrusive fingering current. Due to the complex interactions between driving force, gravity, and inertia, the intrusion current fluctuates at the neutral buoyancy layer. In this study, the separation depth $h_{s}$ is defined as the depth where the gravity current leaves the slope (see Figure 11). The separation depth is important in terms of engineering perspectives as it determines the point where the current stops acting on the artificial structures beneath. The non-dimensional separation depth $H_{s}$ is given by

$$
H_{s}=\frac{h_{s}}{h_{0}}
$$

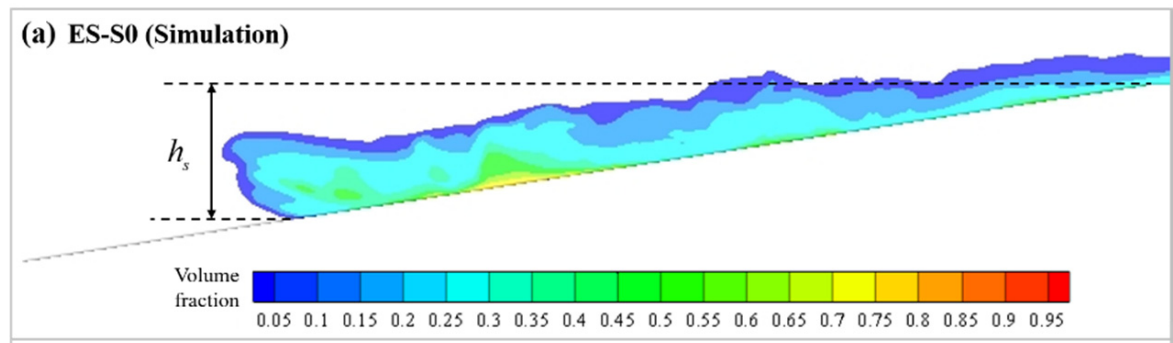

(b) ES-S0 (Experiment)

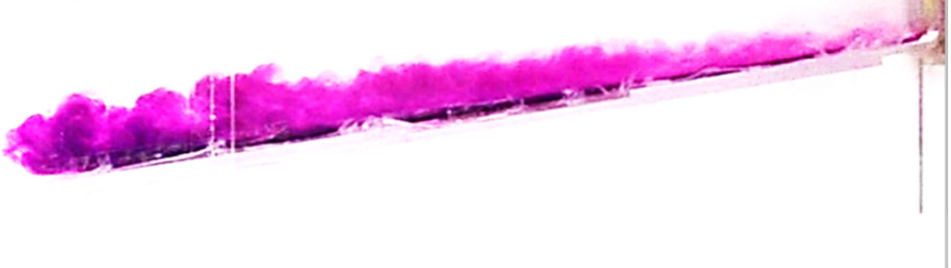

Figure 11. The separation depth of gravity current (Run ES-S0): (a) simulation; (b) experiment.

Figure 12 shows that, in stratified environments, the separation depth of the gravity current obtained from numerical simulations is similar to the experimental measurements. In addition, both numerical simulations and laboratory measurements reveal that the separation depth is inversely proportional to the density of vegetation patches. This is because, as the vegetation density increases, the generated wakes behind vegetation stems increase local entrainment and mixing and cause dense fluid trapped between vegetation stems. The density of the current flowing out from vegetation decreases and it reaches the neutral buoyancy level of ambient fluids earlier, i.e., the current separates from the slope at a shallower depth and horizontally intrudes into the stratified environments. 


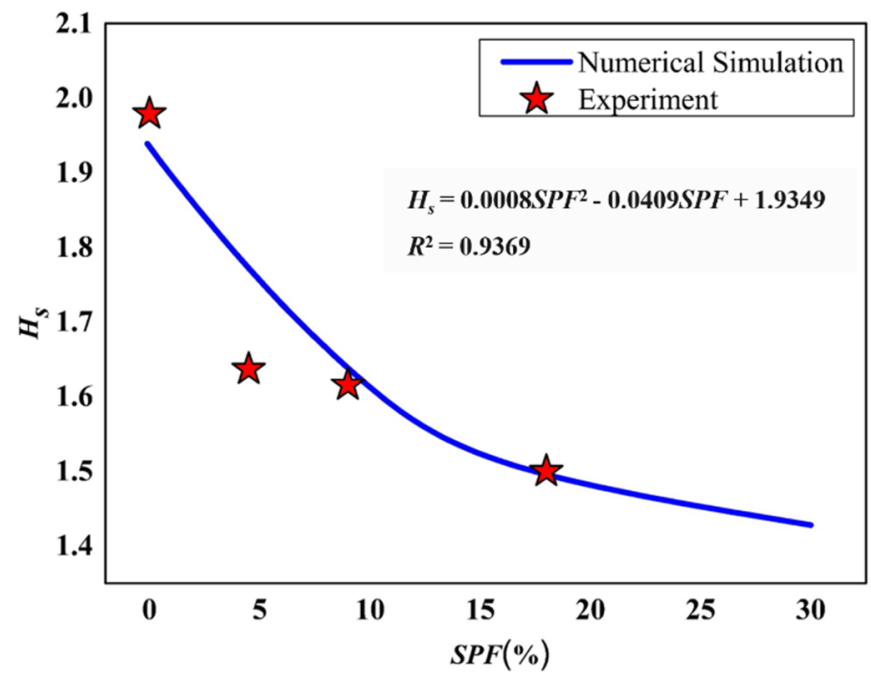

Figure 12. Lateral averaged dimensionless separation depth of the gravity current flowing through vegetation patches with different densities. The numerical results are presented in the form of the fitting curve with the coefficient of determination $R^{2}=0.9369$.

\section{Conclusions}

In this study, the LES turbulent model combined with the mixture model was used to investigate the impact of emergent and rigid vegetation on the dynamics of downslope gravity currents in stratified environments. Morphological patterns, propagation processes, velocity profile, entrainment coefficient, and separation depth were our main foci. Laboratory experiments were also carried out to assess the reliability of the numerical model, and satisfying agreement between the numerical results and the laboratory measurements was found. The main conclusions are drawn as follows.

(1) The dense fluids blocked in front of vegetation patches mix with local ambient fluid, leading to the locally elevated K-H instability at the interface of current-ambient fluid. The presence of vegetation cylinders also leads to severe lateral non-uniformity of the current front, causing more evident lobes and clefts of the current head structure.

(2) In stratified environments, emergent vegetation reduces the current velocity, and the level of velocity reduction is proportional to the vegetation density due to greater vegetation drag. In addition, the transition point of current velocity, i.e., from the acceleration to deceleration phases, appears earlier as the vegetation becomes dense.

(3) In stratified environments, initial $E_{\text {buik }}$ values are larger than their counterparts in homogeneous environments. The peak value of $E_{b u i k}$ is inversely proportional to the vegetation density, while the final converged value of $E_{\text {buik }}$ is proportional to the vegetation density. The vegetation patches make the degree of fluctuation of the $E_{\text {inst }}$ more intense, and even negative values appear locally, indicating that the gravity current is detrained into the ambient fluid.

(4) Both numerical simulations and laboratory measurements reveal that the separation depth is inversely proportional to the density of vegetation patches. As the vegetation density increases, the generated wakes behind vegetation stems increase local entrainment and mixing and cause dense fluid trapped between vegetation stems. The density of the current flowing out from vegetation decreases and it reaches the neutral buoyancy level of ambient fluids earlier, i.e., the current separates from the slope at a shallower depth and horizontally intrudes into the stratified environment. Once the separation depth is varied, the point where gravity currents stop destroying artificial structures and the positions of biological hotspots will be altered. Therefore, placing these rigid and emergent vegetation patches (also called obstacles) in nearshore regions could become an effective engineering measure to prevent the destruction of underwater structures and change ecological environments. In addition, the relevant 
results of the article can also provide a scientific basis for the construction of green engineering and disaster prevention and mitigation in nearshore areas.

Author Contributions: Conceptualization, Y.-J.C. and Y.-T.L.; Methodology, Y.-J.C. and Y.-T.L.; Laboratory Measurement, Y.-Q.Y. and D.-R.H.; Data Analysis, Y.-Q.Y. and D.-R.H.; Software, Y.-Q.Y. and D.-R.H.; Writing-Original Draft Preparation, Y.-T.L., Y.-Q.Y. and D.-R.H.; Writing-Review and Editing, Y.-J.C.; Funding Acquisition, Y.-J.C. and Y.-T.L. All authors have read and agreed to the published version of the manuscript.

Funding: This research was funded by the Ministry of Science and Technology of Taiwan (Grant no. MOST 110-2221-E-019-084); the National Natural Science Foundation of China (Grant no. 41876089); Zhejiang Provincial Natural Science Foundation of China (Grant no. LY20A020009).

Institutional Review Board Statement: Not applicable.

Informed Consent Statement: Not applicable.

Data Availability Statement: All data generated or analyzed during this study are included in this article.

Conflicts of Interest: The authors declare no conflict of interest.

\section{References}

1. Simpson, E. Gravity Currents: In the Environment and the Laboratory; Cambridge University Press: Cambridge, UK, 1999.

2. Ho, H.-C.; Lin, Y.-T. Gravity currents over a rigid and emergent vegetated slope. Adv. Water Resour. 2015, 76, 72-80. [CrossRef]

3. Marleau, L.J.; Flynn, M.R.; Sutherland, B.R. Gravity currents propagating up a slope in a two-layer fluid. Phys. Fluids 2015, 27, 036601. [CrossRef]

4. Dai, A. Non-Boussinesq gravity currents propagating on different bottom slopes. J. Fluid Mech. 2014, 741, 658-680. [CrossRef]

5. He, Z.; Zhao, L.; Lin, T.; Hu, P.; Lv, Y.; Ho, H.C.; Lin, Y.T. Hydrodynamics of gravity currents down a ramp in linearly stratified environments. J. Hydraul. Eng. 2016, 143, 04016085. [CrossRef]

6. He, Z.; Zhao, L.; Zhu, R.; Hu, P. Separation of particle-laden gravity currents down a slope in linearly stratified environments. Phys. Fluids 2019, 31, 106602. [CrossRef]

7. Martin, A.; Negretti, M.E.; Ungarish, M.; Zemach, T. Propagation of a continuously supplied gravity current head down bottom slopes. Phys. Rev. Fluids 2020, 5, 054804. [CrossRef]

8. Dai, A.; Huang, Y.-L. Experiments on gravity currents propagating on unbounded uniform slopes. Environ. Fluid Mech. 2020, 20, 1637-1662. [CrossRef]

9. Snow, K.; Sutherland, B.R. Particle-laden flow down a slope in uniform stratification. J. Fluid Mech. 2014, 755, 251-273. [CrossRef]

10. Guo, Y.; Zhang, Z.; Shi, B. Numerical simulation of gravity current descending a slope into a linearly stratified environment. J. Hydraul. Eng. 2014, 140, 04014061. [CrossRef]

11. Testik, F.Y.; Yilmaz, N.A. Anatomy and propagation dynamics of continuous-flux release bottom gravity currents through emergent aquatic vegetation. Phys. Fluids 2015, 27, 056603. [CrossRef]

12. Zhou, J.; Venayagamoorthy, S.K. Numerical simulations of intrusive gravity currents interacting with a bottom-mounted obstacle in a continuously stratified ambient. Environ. Fluid Mech. 2017, 17, 191-209. [CrossRef]

13. Tanino, Y.; Nepf, H.M. Laboratory investigation of mean drag in a random array of rigid, emergent cylinders. J. Hydraul. Eng. 2008, 134, 34-41. [CrossRef]

14. Tanino, Y.; Nepf, H.M.; Kulis, P.S. Gravity currents in aquatic canopies. Water Resour. Res. 2005, 41, W12402. [CrossRef]

15. Gu, Z.; Ho, H.C.; Wang, Z.; Lin, Y.T. Laboratory Studies on Nearshore Density-Driven Exchange Flow over a Partly Vegetated Slope. Water 2018, 10, 1073. [CrossRef]

16. Zhou, J.; Venayagamoorthy, S.K. Impact of ambient stable stratification on gravity currents propagating over a submerged canopy. J. Fluid Mech. 2020, 898, A15. [CrossRef]

17. Han, D.R.; Xiong, J.; Xie, X.Y. Effects of emergent and submerged rigid vegetation configurations on gravity current dynamics. Effects of emergent and submerged rigid vegetation configurations on gravity current dynamics. Environ. Fluid Mech. 2021, 21, 1165-1187. [CrossRef]

18. Ungarish, M. On gravity currents in a linearly stratified ambient: A generalization of Benjamin's steady-state propagation results. J. Fluid Mech. 2006, 548, 49. [CrossRef]

19. Johnson, G.; Massoudi, M.; Rajagopal, K.R. Flow of a fluid-Solid mixture between flat plates. Chem. Eng. Sci. 1991, 46, 1713-1723. [CrossRef]

20. Tokyay, T.E.; García, M.H. Effect of initial excess density and discharge on constant flux gravity currents propagating on a slope. Environ. Fluid Mech. 2013, 14, 409-429. [CrossRef]

21. Wang, M.; Avital, E.; Korakianitis, T.; Williams, J.; Ai, K. A numerical study on the influence of curvature ratio and vegetation density on a partially vegetated U-bend channel flow. Adv. Water Resour. 2021, 148, 103843. [CrossRef] 
22. Liu, M.; Huai, W.; Ji, B.; Han, P. Numerical study on the drag characteristics of rigid submerged vegetation patches. Phys. Fluids 2021, 33, 085123. [CrossRef]

23. Yuksel-Ozan, A.; Constantinescu, G. Front velocity and structure of bottom gravity currents with a low volume of release propagating in a porous medium. Environ. Fluid Mech. 2016, 18, 241-265. [CrossRef]

24. Ottolenghi, L.; Adduce, C.; Inghilesi, R.; Armenio, V.; Roman, F. Entrainment and mixing in unsteady gravity currents. J. Hydraul. Res. 2016, 54, 541-557. [CrossRef]

25. Altinakar, M.S.; Graf, W.H.; Hopfinger, E.J. Flow structure in turbidity currents. J. Hydraul. Res. 1996, 34, 713-718. [CrossRef]

26. Yang, Y.; Lin, Y.T.; Ji, X.Y. Hydrodynamic characteristics of flow over emergent vegetation in a strongly curved channel. J. Hydraul. Res. 2021. [CrossRef] 